\title{
Multi-recipient Public-Key Encryption with Shortened Ciphertext
}

\author{
Kaoru Kurosawa \\ Department of Computer and Information Sciences, Ibaraki University, \\ 4-12-1 Nakanarusawa, Hitachi, Ibaraki, 316-8511, Japan \\ Tel/Fax. +81-294-38-5135 \\ kurosawa@cis.ibaraki.ac.jp
}

\begin{abstract}
In the trivial $n$-recipient public-key encryption scheme, a ciphertext is a concatenation of independently encrypted messages for $n$ recipients. In this paper, we say that an $n$-recipient scheme has a "shortened ciphertext" property if the length of the ciphertext is almost a half (or less) of the trivial scheme and the security is still almost the same as the underlying single-recipient scheme. We first present (multi-plaintext, multi-recipient) schemes with the "shortened ciphertext" property for ElGamal scheme and Cramer-Shoup scheme. We next show (single-plaintext, multi-recipient) hybrid encryption schemes with the "shortened ciphertext" property.
\end{abstract}

\section{Introduction}

\section{$1.1 \quad$ Background}

Suppose that there are $n$ recipients. Let $p k_{i}$ be the public key of recipient $i$ for $1 \leq i \leq n$. The security of a public-key encryption scheme in the multi-recipient setting is different from the single-recipient setting. For example, if $e$ is the common public exponent in RSA, then e encryptions of the same plaintext $M$ under different moduli lead to an easy recovery of $M$. Further results by Hastad [11 and Coppersmith $7 / 8$ proved that even the time-stamp variants can be successfully attacked with $e$ ciphertexts.

In the trivial $n$-recipient public-key encryption scheme, a ciphertext is just a concatenation of independently encrypted messages for $n$ recipients using a single-recipient public-key encryption algorithm $\mathcal{E}$. That is, $\mathcal{E}_{p k_{1}}\left(M_{1}\right) \| \cdots$ $\| \mathcal{E}_{p k_{n}}\left(M_{n}\right)$, where $\|$ denotes concatenation. In general, this trivial scheme is not secure in the sense of invertibility even if $\mathcal{E}$ is secure in the same sense, as shown in the above RSA example.

Recently, Bellare et al. [2] and Baudron et al. [1] independently proved that the trivial $n$-recipient scheme is secure in the sense of indistinguishability [10] if $\mathcal{E}$ is secure in the same sense, where indistinguishability is a stronger security notion than invertibility.

However, their nice results 21] still do not capture the essence of the multirecipient setting:

D. Naccache and P. Paillier (Eds.): PKC 2002, LNCS 2274, pp. 4863 2002

(C) Springer-Verlag Berlin Heidelberg 2002 
(1) The length of the ciphertext of the trivial $n$-recipient scheme is $n$ times larger than that of the underlying single-recipient scheme.

(2) Consider a single-recipient hybrid encryption scheme which encrypts a long message $M$ using a pseudorandom generator $G$ and sends the seed $r$ of $G$ using a public-encryption scheme. That is,

$$
C=M \oplus G(r) \| \mathcal{E}_{p k}(r),
$$

where || denotes concatenation. A natural extension of the hybrid scheme to an $n$-recipient scheme will be that

$$
M \oplus G(r)\left\|\mathcal{E}_{p k_{1}}(r)\right\| \cdots \| \mathcal{E}_{p k_{n}}(r) .
$$

Their results 21] only imply that the latter part $\mathcal{E}_{p k_{1}}(r)\|\cdots\| \mathcal{E}_{p k_{n}}(r)$ is secure in the sense of indistinguishability if the single-recipient part $\mathcal{E}_{p k}(r)$ is secure in the same sense.

\subsection{Our Contribution}

In this paper, we consider $n$-recipient public-key encryption schemes such that the length of the ciphertext is almost a half (or less) of the trivial $n$-recipient scheme and the security is still almost the same as the underlying single-recipient scheme. We say that such a scheme has a "shortened ciphertext" property.

1. We first give the definitions of our model and the security.

2. We next present (multi-plaintext, multi-recipient) schemes with the "shortened ciphertext" property for ElGamal scheme and Cramer-Shoup scheme and prove their security.

3. We also prove that the above mentioned (single-plaintext, multi-recipient) scheme of eq.(2) is secure in the sense of indistinguishability against chosen plaintext attack if the underlying single-recipient public-key scheme is secure in the same sense.

4. We finally present how to construct a (single-plaintext, multi-recipient) scheme secure against chosen ciphertext attack with the "shortened ciphertext" property. The underlying single-recipient public-key scheme needs to be secure in the sense of indistinguishability against chosen ciphertext attack. (For example, we can use Rabin-SAEP or RSA-SAEP ${ }^{+}$[4] as the underlying single-recipient scheme.)

Cramer-Shoup scheme is a practical public-key encryption scheme which is secure in the sense of indistinguishability against chosen-ciphertext attack under the decision Diffie-Hellman (DDH) assumption in the standard model 9]. The basic Cramer-Shoup scheme uses universal one-way hash functions (UOH) 9, Sec.3]. Bellare et al. derived the concrete security of the basic Cramer-Shoup scheme by assuming the concrete security of UOH [2]. On the other hand, Cramer and Shoup also presented a hash-free variant which does not use UOH [9, Sec.5.3].

We derive the concrete security of the hash-free variant of Cramer-Shoup scheme. It is of independent interest because it truly depends only on the DDH 
assumption, but not UOH. We then present a (multi-plaintext, multi-recipient) hash-free Cramer-Shoup scheme that has the "shortened ciphertext" property.

One further advantage of our multi-recipient schemes (in the discrete log setting) is that the encryption operation can be significantly faster than if the encryption operations were performed separately for each recipient.

Finally, in all of our multi-recipient schemes, the decryption algorithm is the same as the single-recipient one. Therefore, no extra cost is required for each recipient.

\subsection{Related Works}

The "broadcast" problem has been addressed by other authors in the context of traitor-tracing [612513. The traitor-tracing schemes such that 12513] can have even shorter ciphertexts than our schemes, but with the tradeoff that a small coalition of recipients can break the traitor-tracing aspect of the scheme, i.e., construct a new private key that does not identify anyone in the coalition. In our schemes, no coalition can do this since each private key uniquely identifies the recipient.

Bellare and Rogaway 3 proved that the single recipient hybrid encryption scheme shown in eq.(1) is secure in the sense of indistinguishability against chosen plaintext under the random oracle moel if $\mathcal{E}_{p k}$ is a trapdoor oneway permutation. They also proved that the following scheme secure in the sense of indistinguishability against chosen ciphertext under the random oracle moel.

$$
C=\mathcal{E}_{p k}(r)\|M \oplus G(r)\| H(M \| r),
$$

where $H$ is a hash function. Before that, Zheng and Seberry [16] proposed a scheme such that

$$
C=\mathcal{E}_{p k}(r) \|(G(r) \oplus(M \| H(M))
$$

\section{Single-Recipient Encryption Scheme}

A single-recipient public-key encryption scheme $\mathcal{P} \mathcal{E}=(\mathcal{K}, \mathcal{E}, \mathcal{D})$ consists of three algorithms. The key generation algorithm $\mathcal{K}$ outputs $(p k, s k)$ on input some global information $I$, where $p k$ is a public key and $s k$ is the secret key; we write $(p k, s k) \stackrel{R}{\leftarrow} \mathcal{K}(I)$. The encryption algorithm $\mathcal{E}$ outputs a ciphertext $C$ on input the public key $p k$ and a plaintext $M$; we write $C \stackrel{R}{\leftarrow} \mathcal{E}_{p k}(M)$. The decryption algorithm $\mathcal{D}$ outputs $M$ or reject on input the secret key $s k$ and a ciphertext $C$; we write $x \leftarrow \mathcal{D}_{s k}(C)$, where $x=M$ or reject. We require that $\mathcal{D}_{s k}\left(\mathcal{E}_{p k}(M)\right)=M$ for each plaintext $M$.

An adversary $B$ runs in two stages. In "find" stage, it takes a public key $p k$ and outputs two equal length messages $M_{0}$ and $M_{1}$ together with some state information state. In "guess" stage, it gets a challenge ciphertext $C_{b} \stackrel{R}{\leftarrow} \mathcal{E}_{p k}\left(M_{b}\right)$ from the encryption oracle $\mathcal{E}_{p k}$, where $b$ is a randomly chosen bit. $B$ finally outputs a bit $\tilde{b}$. The advantage of $B$ is measured by the probability $\operatorname{Pr}(\tilde{b}=b)$. 
Formally, the security of $\mathcal{P E}$ in the sense of indistinguishability against chosen-plaintext attack is defined as follows.

Definition 2.1. For $b=0$ and 1 , define the experiment as follows.

$$
\begin{gathered}
\left(p k_{1}, s k_{1}\right) \stackrel{R}{\longleftarrow} K(I),\left(M_{0}, M_{1}, \text { state }\right) \stackrel{R}{\leftarrow} B(\text { find }, p k), \\
C_{b} \stackrel{R}{\longleftarrow} \mathcal{E}_{p k}\left(M_{b}\right), \tilde{b} \stackrel{R}{\longleftarrow} B\left(\text { guess }, C_{b}, \text { state }\right) .
\end{gathered}
$$

Let

$$
\begin{aligned}
\operatorname{Adv}_{\mathcal{P} \mathcal{E}, I}^{s-c p a}(B) & \stackrel{\text { def }}{=} \operatorname{Pr}(\tilde{b}=0 \mid b=0)-\operatorname{Pr}(\tilde{b}=0 \mid b=1) \\
\operatorname{Adv}_{\mathcal{P} \mathcal{E}, I}^{s-c p a}(t) & \stackrel{\text { def }}{=} \max _{B} \operatorname{Adv}_{\mathcal{P} \mathcal{E}, I}^{s-c p a}(B),
\end{aligned}
$$

where the maximum is over all $B$ with time-complexity $t$.

(In the superscript, $s$ - denotes "single recipient".)

Definition 2.2. We say that $\mathcal{P} \mathcal{E}$ is secure against chosen-plaintext attack if $\operatorname{Adv}_{\mathcal{P} \mathcal{E}, I}^{s-p a}(t)$ is negligible for polynomially bounded $t$, where the complexity is measured as a function of a security parameter.

It is easy to see that

$$
\operatorname{Pr}(\tilde{b}=b)=\frac{1}{2}+\frac{1}{2} \operatorname{Adv}_{\mathcal{P} \mathcal{E}, I}^{\mathrm{s}, \mathrm{cpa}}(B)
$$

The security against chosen-ciphertext attack is defined similarly except for that the adversary $B$ gets the decryption oracle $\mathcal{D}_{s k}$ and is allowed to query any ciphertext $C$ at most $q_{d}$ times, where it must be that $C \neq C_{b}$ in the guess stage. We denote the advantages by $\operatorname{Adv}_{\mathcal{P} \mathcal{E}, I}^{\text {s-cca }}(B)$ and $\operatorname{Adv}_{\mathcal{P} \mathcal{E}, I}^{\text {s-cca }}\left(t, q_{d}\right)$, respectively.

\section{Multi-recipient Encryption Scheme}

Suppose that there are $n$ recipients. Let $N \stackrel{\text { def }}{=}\{1, \cdots, n\}$. We define (singleplaintext, multi-recipient) public-key encryption schemes and (multi-plaintext, multi-recipient) public-key encryption schemes as follows.

- In a (single-plaintext, multi-recipient) public-key encryption scheme, a sender sends the same plaintext $M$ secretly to a subset of recipients $S \subseteq N$ by broadcasting a ciphertext $C_{S}$.

- In a (multi-plaintext, multi-recipient) public-key encryption scheme, a sender sends an independent plaintext $M_{i}$ secretly to each recipient $i \in S$ by broadcasting a ciphertext $C_{S}$. 


\section{1 "Shortened Ciphertext" Property}

A multi-recipient public-key encryption scheme is naturally constructed from a single-recipient public-key encryption scheme $\mathcal{P} \mathcal{E}=(\mathcal{K}, \mathcal{E}, \mathcal{D})$ as follows. The key generation algorithm runs $\mathcal{K}(I) n$ times independently. A ciphertext $C_{N}$ is

$$
C_{N}=\mathcal{E}_{p k_{1}}\left(M_{1}\right)\|\cdots\| \mathcal{E}_{p k_{n}}\left(M_{n}\right)
$$

where || denotes concatenation. We call this scheme the trivial multi-recipient scheme.

Bellare et al. 2 proved that the trivial multi-recipient scheme is secure in the sense of indistinguishability if $\mathcal{P} \mathcal{E}$ is secure in the same sense. Baudron et al.

11 proved the same result independently. However, the length of the ciphertext of the trivial multi-recipient scheme is $n$ times larger than that of the singlerecipient scheme.

In this paper, we consider multi-recipient public-key encryption schemes such that (1) the length of the ciphertext is almost a half (or less) of the trivial multirecipient scheme and (2) the security is still almost the same as the underlying single-recipient scheme. We say that such a scheme has a "shortened ciphertext" property.

\subsection{Our Model}

For a single-recipient public-key encryption scheme $\mathcal{P} \mathcal{E}=(\mathcal{K}, \mathcal{E}, \mathcal{D})$, we define a (multi-plaintext, multi-recipient) public-key encryption scheme $\mathcal{P} \mathcal{E}^{n}=$ $\left(\mathcal{K}^{n}, \mathcal{E}^{n}, T A K E\right)$ as follows.

- The key generation algorithm $\mathcal{K}^{n}$ outputs $p k \stackrel{\text { def }}{=}\left(p k_{1}, \cdots, p k_{n}\right)$ and $\underline{s k} \stackrel{\text { def }}{=}$ $\left(s k_{1}, \cdots, s k_{n}\right)$ on input some global information $I$, where $\left(p k_{i}, s k_{i}\right)$ is a pair of encryption/decryption keys of recipient $i$.

- For $S=\left\{1_{1}, \cdots i_{s}\right\}$, let $M_{i_{j}}$ be a plaintext for recipient $i_{j} \in S$. Let $\underline{M}_{S} \stackrel{\text { def }}{=}$ $\left(M_{i_{1}}, \cdots, M_{i_{s}}\right)$. Then the encryption algorithm $\mathcal{E}^{n}$ computes a ciphertext $C_{S}$ for $\underline{M}_{S}$ on input $\underline{p k}, S$ and $\underline{M}_{S}$; we write $C_{S} \stackrel{R}{\leftarrow} \mathcal{E}_{p k}^{n}\left(S, \underline{M}_{S}\right)$.

$-T A K E$ is a hash function that takes a part of a ciphertext as follows. For $T \subset$ $S \subseteq N$, it outputs $C_{T}$ on input $T, S$ and $C_{S}$. We write $C_{T} \leftarrow T A K E_{T}\left(C_{S}\right)$. Especially, for $i \in S$, we write $C_{i} \leftarrow T A K E_{i}\left(C_{S}\right)$.

We require that $\mathcal{D}_{s k_{i}}\left(T A K E_{i}\left(C_{S}\right)\right)=M_{i}$ for all $i \in S$ and any $M_{i}$.

A (single-plaintext, multi-recipient) public-key encryption scheme is defined similarly.

Remark 3.1. In our multi-recipient schemes, the decryption algorithm is the same as the single-recipient scheme. Therefore, no extra cost is required for each recipient. 


\subsection{Security}

We generalize the definition of security for the multi-recipient setting given by Bellare et al. 2] to (multi-plaintext,multi-recipient) schemes as follows.

We consider an experiment as follows. At the beginning, a challenge bit $b$ is randomly chosen and fixed. An adversary $B$ is provided with the encryption oracle $\mathcal{E}_{\underline{p k}}^{n}$ and it is allowed to query $\left(S, \underline{M}_{S}^{0}, \underline{M}_{S}^{1}\right)$ at most $q_{e}$ times. $\mathcal{E}_{\underline{p k}}^{n}$ returns a ciphertext $\mathcal{E}_{\underline{p k}}^{n}\left(S, \underline{M}_{S}^{b}\right.$ ). (Since $b$ is fixed at the beginning, the same $b$ is used across all the queries.) $B$ finally outputs a bit $\tilde{b}$. We require that $\left|M_{i_{j}}^{0}\right|=\left|M_{i_{j}}^{1}\right|$ for all $i_{j} \in S$, where $\underline{M}_{S}^{0}=\left(M_{i_{1}}^{0}, \cdots, M_{i_{s}}^{0}\right)$ and $\underline{M}_{S}^{1}=\left(M_{i_{1}}^{1}, \cdots, M_{i_{s}}^{1}\right)$.

Each time, $B$ can choose $\left(S, \underline{M}_{S}^{0}, \underline{M}_{S}^{1}\right)$ arbitrarily, where $S$ as well as $\left(\underline{M}_{S}^{0}, \underline{M}_{S}^{1}\right)$ may be related to his other queries to $\mathcal{E}_{p k}^{n}$. Then the security of $\mathcal{P} \mathcal{E}^{n}$ against chosen-plaintext attack is defined as follows.

Definition 3.1. For $b=0$ and 1 , define the experiment as follows.

$$
\left.(\underline{p k}, \underline{s k}) \stackrel{R}{\leftarrow} K^{n}(I), \tilde{b} \leftarrow B^{\mathcal{E}^{n}} \stackrel{(I,}{p k}\right) .
$$

Let

$$
\begin{aligned}
& \operatorname{Adv}_{\mathcal{P E}^{n}, I}^{n-c p a}(B) \stackrel{\text { def }}{=} \operatorname{Pr}(\tilde{b}=0 \mid b=0)-\operatorname{Pr}(\tilde{b}=0 \mid b=1) \\
& \operatorname{Adv}_{\mathcal{P} \mathcal{E}^{n}, I}^{n-c p a}\left(t, q_{e}\right) \stackrel{\text { def }}{=} \max _{B} \operatorname{Adv}_{\mathcal{P} \mathcal{E}^{n}, I}^{n-c p a}(B),
\end{aligned}
$$

where the maximum is over all B with time-complexity $t$.

In the superscript, $n$ - denotes " $n$ recipients".

Definition 3.2. We say that $\mathcal{P} \mathcal{E}^{n}$ is secure against chosen-plaintext attack if $\operatorname{Adv}_{\mathcal{P} \mathcal{E}^{n}, I}^{n-c p a}(t)$ is negligible for polynomially bounded $t$, where the complexity is measured as a function of a security parameter.

The security against chosen-ciphertext attack is defined similarly except for that the adversary $B$ gets $n$ decryption oracles $\mathcal{D}_{s k_{1}}, \cdots, \mathcal{D}_{s k_{n}}$. It is allowed to query any ciphertext $C$ to any decryption oracle $\mathcal{D}_{s k_{i}}$ at most $q_{d}$ times for each $i$, where it must be that $C \neq T A K E_{i}\left(C_{S}\right)$ for any output $C_{S}$ of the encryption oracle $\mathcal{E}_{x p k}$. We denote the advantages by $\operatorname{Adv}_{\mathcal{P} \mathcal{E}^{n}, I}^{n-\text { cca }}(B)$ and $\operatorname{Adv}_{\mathcal{P} \mathcal{E}^{n}, I}^{n \text {-cca }}\left(t, q_{e}, q_{d}\right)$, respectively.

The security of (single-plaintext, multi-recipient) schemes is defined similarly. For simplicity, the same notation as above will be used.

Remark 3.2. In the definition of Bellare et al. [2, (i) $|S|=1$ and there are $n$ encryption oracles $\mathcal{E}_{p k_{1}}, \cdots, \mathcal{E}_{p k_{n}}$. (ii) $B$ is allowed to query at most $q_{e}$ times to each $\mathcal{E}_{p k_{i}}$. It is easy to see that our definition is more general if we ignore (ii). 


\subsection{Sufficient Condition}

We say that an adversary is type 0 if $q_{e}=1$ and his query to $\mathcal{E}_{p k}^{n}$ is $\left(N, \underline{M}_{N}^{0}, \underline{M}_{N}^{1}\right)$. That is, we consider an adversary which runs in two stages, the find stage and the guess stage, as in the single-recipient case.

Definition 3.3. Let $\operatorname{AdvTO}_{\mathcal{P} \mathcal{E}^{n}, I}^{n-c p a}(t)$ be the $\max _{B} \operatorname{Adv}_{\mathcal{P E}^{n}, I}^{n-c p a}(B)$, where the maximum is over all type 0 adversaries $B$ with time-complexity $t$. Define $\operatorname{AdvTO}_{\mathcal{P E}^{n}, I}^{n-c c a}\left(t, q_{d}\right)$ similarly.

The next lemma shows that $\mathcal{P} \mathcal{E}^{n}$ is secure if $\operatorname{AdvTO}_{\mathcal{P} \mathcal{E}^{n}, I}^{n-x}(t)$ is negligible, where $x=c p a$ or cca. Therefore, we do not have to evaluate $\operatorname{Adv}_{\mathcal{P}_{\mathcal{E}}^{n}, I}^{n-\mathrm{x}}\left(t, q_{e}\right)$ directly.

Let $T_{n}$ denote the time to compute a ciphertext $C_{N}=\mathcal{E}_{\underline{p k}}^{n}\left(N, \underline{M}_{N}\right)$.

Lemma 3.1. In an $n$-recipient broadcast/multicast public-key encryption scheme $\mathcal{P} \mathcal{E}^{n}$

$$
\begin{aligned}
\operatorname{Adv}_{\mathcal{P} \mathcal{E}^{n}, I}^{n-c p a}\left(t, q_{e}\right) & \leq q_{e} \cdot \operatorname{AdvTO}_{\mathcal{P} \mathcal{E}^{n}, I}^{n-c p a}\left(t^{\prime}\right), \\
\operatorname{Adv}_{\mathcal{P} \mathcal{E}^{n}, I}^{n-c c a}\left(t, q_{e}, q_{d}\right) & \leq q_{e} \cdot \operatorname{AdvTO}_{\mathcal{P} \mathcal{E}^{n}, I}^{n-c c a}\left(t^{\prime}, q_{d}\right),
\end{aligned}
$$

where $t^{\prime}=t+O\left(q_{e} T_{n}\right)$

A proof is given in Appendix.

\section{Multi-recipient "ElGamal" Encryption Scheme}

In this section, we show a (multi-plaintext, multi-recipient) ElGamal scheme which has the "shortened ciphertext" property. Let $\mathcal{G}$ be a group with a prime order $p$ and let $g$ be a generator of $\mathcal{G}$. Let $I=(p, g)$ be the global information.

Let $T^{\exp }$ denote the time needed to perform an exponentiation in $\mathcal{G}$.

\subsection{ElGamal Scheme and DDH Problem}

Informally, the decision Diffie-Hellman (DDH) problem is stated as follows. Given $g^{x}, g^{y}, g^{z}$, decide if $z=x y \bmod p$ with nonnegligible probability. Formally, let

$$
\begin{aligned}
& D H \stackrel{\text { def }}{=}\left\{\left(g^{x}, g^{y}, g^{x y}\right) \mid x \in Z_{p}, y \in Z_{p}\right\} \\
& R A \stackrel{\text { def }}{=}\left\{\left(g^{x}, g^{y}, g^{z}\right) \mid x \in Z_{p}, y \in Z_{p}, z \in Z_{p}\right\} .
\end{aligned}
$$

Let $D$ be a distinguisher which outputs 0 or 1 . Define

$$
\begin{aligned}
\operatorname{Adv}_{p, g}^{d d h}(D) & \stackrel{\text { def }}{=} \operatorname{Pr}[D(X)=0 \mid X \in D H]-\operatorname{Pr}[D(X)=0 \mid X \in R A], \\
\operatorname{Adv}_{p, g}^{d d h}(t) & \stackrel{\text { def }}{=} \max _{D} \operatorname{Adv}_{p, g}(D),
\end{aligned}
$$

where the maximum is over all $D$ with "time-complexity" $t$. The DDH assumption is that $\operatorname{Adv}_{p, g}^{d d h}(t)$ is negligible. 
ElGamal encryption scheme $\mathcal{E} \mathcal{G}=(\mathcal{K}, \mathcal{E}, \mathcal{D})$ is as follows.

$$
\begin{aligned}
\mathcal{K}(I) & : s k=x, p k=X\left(\leftarrow g^{x}\right), \text { where } x \stackrel{R}{\leftarrow} Z_{p} . \\
\mathcal{E}_{I, X}(M) & :(Y, W)=\left(g^{r}, M \cdot X^{r}\right), \text { where } r \stackrel{R}{\leftarrow} Z_{p} . \\
\mathcal{D}_{I, x}(Y, W) & : M \leftarrow W \cdot Y^{-x} .
\end{aligned}
$$

It is well known that ElGamal scheme is secure in the sense of indistinguishability against chosen plaintext attack under the DDH assumption.

\subsection{Proposed Scheme}

Now we present the proposed (multi-plaintext,multi-recipient) ElGamal scheme $\mathcal{E G}^{n}=\left(\mathcal{K}^{n}, \mathcal{E}^{n}, T A K E\right)$. The key generation algorithm $\mathcal{K}^{n}(I)$ runs $\mathcal{K}(I) n$ times independently. Let $x_{i}$ be the secret key and $X_{i}\left(=g^{x_{i}}\right)$ be the public-key of recipient $i$.

For $S=\left\{1_{1}, \cdots i_{s}\right\}$, let $M_{i_{j}}$ be a plaintext for recipient $i_{j} \in S$. Then a ciphertext for $S$ is

$$
C_{S}=\left(g^{r}, M_{i_{1}} X_{i_{1}}^{r}, \ldots, M_{i_{s}} X_{i_{s}}^{r}\right),
$$

where $r \stackrel{R}{\leftarrow} Z_{p}$. TAKE $E_{i}$ is defined as $\left(g^{r}, M_{i} X_{i}^{r}\right) \leftarrow T A K E_{i}\left(C_{S}\right)$. For $T \subset S \subseteq$ $N, C_{T} \leftarrow T A K E_{T}\left(C_{S}\right)$ is defined naturally.

We will show that our scheme has the "shortened ciphertext" property. First, in the trivial multi-recipient scheme, a ciphertext is

$$
C_{S}^{\text {trivial }}=\left(g^{r_{i_{1}}}, M_{i_{1}} X_{1}^{r_{i_{1}}}\right)\|\cdots\|\left(g^{r_{i_{s}}}, M_{s} X_{s}^{r_{i_{s}}}\right)
$$

Therefore, in our scheme, the size of the ciphertext is almost a half of that of the trivial multi-recipient scheme. We next prove that our scheme is still secure. More precisely, we prove that our scheme is secure in the sense of indistinguishability against chosen plaintext attack under the DDH assumption.

Lemma 4.1. In the proposed (multi-plaintext,multi-recipient) ElGamal encryption scheme,

$$
\operatorname{AdvTO}_{\mathcal{E} \mathcal{G}^{n}(p, g)}^{n-c p a}(t) \leq 2 \cdot \operatorname{Adv}_{p, g}^{d d h}\left(t^{\prime}\right)+\frac{1}{p},
$$

where $t^{\prime}=t+O\left(n \cdot T^{e x p}\right)$.

A proof is given in Appendix. From lemma 4.1 and lemma 3.1, we obtain the following theorem.

Theorem 4.1. In the proposed (multi-plaintext,multi-recipient) ElGamal encryption scheme,

$$
\operatorname{Adv}_{\mathcal{E} \mathcal{G}^{n}(p, g)}^{n-c p a}\left(t, q_{e}\right) \leq q_{e}\left(2 \cdot \operatorname{Adv}_{p, g}^{d d h}\left(t^{\prime}\right)+\frac{1}{p}\right),
$$

where $t^{\prime}=t+O\left(q_{e} n \cdot T^{e x p}\right)$. 
The concrete security of the trivial multi-recipient ElGamal encryption scheme derived by Bellare et al. 2] satisfies the same equation as eq.(4). Hence, the coefficient $q_{e}$ in eq.(5) can be considered as the cost for the "shortened ciphertext" property.

\subsection{S/MIME CMS}

S/MIME CMS (IETF RFC 2630) is a (single-plainext, multi-recipient) scheme such that

$$
C_{S}=\left(g^{r}, W r a p\left(X_{i_{1}}^{r}, K\right), \ldots, W r a p\left(X_{i_{s}}^{r}, K\right)\right),
$$

where $K$ is a content-encryption key to be transported, Wrap is a symmetric key-wrapping operation.

The Wrap operation takes the role of the multiplication in the basic ElGamal scheme. Therefore, Theorem 4.1 shows that this scheme is secure if Wrap is secure enough.

\section{Multi-recipient "Cramer-Shoup" Encryption Scheme}

In this section, we first show the concrete security of the hash-free variant of Cramer-Shoup scheme. We next present a (multi-plaintext,multi-recipient) hashfree Cramer-Shoup scheme which has the "shortened ciphertext" property.

Let $\mathcal{G}$ be a group with a prime order $p$ and let $g_{1}$ be a generator of $\mathcal{G}$. Let $I=\left(p, g_{1}\right)$ be the global information.

\subsection{Concrete Security of the Hash-Free Cramer-Shoup Scheme}

Bellare et al. derived the concrete security of the basic Cramer-Shoup scheme [9. Sec.3] by assuming the security of universal one-way hash functions (UOH) [2]. In this subsection, we derive the concrete security of the hash-free variant of Cramer-Shoup scheme, which does not need to assume UOH.

The hash-free variant of Cramer-Shoup scheme $\mathcal{C S}=(\mathcal{K}, \mathcal{E}, \mathcal{D})$ is as follows [9. Sec.5.3]. Let $F$ be a polynomial time computable injection from $G^{3}$ to $\left(Z_{p}^{*}\right)^{k}$ for some $k$. Let $(p k, s k)$ be

$$
\begin{aligned}
s k: & z, x_{1}, x_{2},\left(y_{11}, y_{12}\right), \cdots,\left(y_{k 1}, y_{k 2}\right), \\
& \text { where each element is randomly taken from } Z_{p} . \\
p k: & g_{2}, h\left(=g_{1}^{z}\right), c\left(=g_{1}^{x_{1}} g_{2}^{x_{2}}\right), d_{1}\left(=g_{1}^{y_{11}} g_{2}^{y_{12}}\right), \cdots, d_{k}\left(=g_{1}^{y_{k 1}} g_{2}^{y_{k 2}}\right), \\
& \text { where } g_{2} \text { is randomly chosen from } G .
\end{aligned}
$$

For a plaintext $M$, let a ciphertext $\left(u_{1}, u_{2}, e, v\right)$ be

$$
u_{1}=g_{1}^{r}, u_{2}=g_{2}^{r}, e=h^{r} M, v=\left(c d_{1}^{\alpha_{1}} \cdots d_{k}^{\alpha_{k}}\right)^{r},
$$

where $r \stackrel{R}{\leftarrow} Z_{p}$ and $\left(\alpha_{1}, \cdots, \alpha_{k}\right)=F\left(u_{1}, u_{2}, e\right)$. 
On input $\left(u_{1}, u_{2}, c, v\right)$, the decryption algorithm $\mathcal{D}_{s k}$ first computes $F\left(u_{1}, u_{2}, e\right)$ $=\left(\alpha_{1}, \cdots, \alpha_{k}\right)$. Next if

$$
v=u_{1}^{x_{1}+\alpha_{1} y_{11}+\cdots+\alpha_{k} y_{k 1}} u_{2}^{x_{2}+\alpha_{1} y_{12}+\cdots+\alpha_{k} y_{k 2},}
$$

Then $\mathcal{D}_{s k}$ outputs

$$
M \leftarrow e / u_{1}^{z}
$$

Otherwise, $\mathcal{D}_{s k}$ outputs reject. Let

$$
\epsilon \stackrel{\text { def }}{=}\left(1-\frac{1}{p}\right) \frac{q_{d}}{p}+\frac{1}{p} .
$$

Theorem 5.1. In the hash-free Cramer-Shoup scheme,

$$
\operatorname{Adv}_{\mathcal{C} \mathcal{S},\left(p, g_{1}\right)}^{s-c c a}\left(t, q_{d}\right) \leq 2 \cdot \operatorname{Adv}_{p, g_{1}}^{d d h}\left(t^{\prime}\right)+3 \epsilon
$$

where $t^{\prime}=t+O\left(q_{d} \cdot T^{e x p}\right)$.

A proof will be given in the final paper.

\subsection{Proposed Scheme}

Now the proposed (multi-plaintext,multi-recipient) hash-free Cramer-Shoup scheme $\mathcal{C S}^{n}=\left(\mathcal{K}^{n}, \mathcal{E}^{n}, T A K E\right)$ is described as follows. The key generation algorithm $\mathcal{K}^{n}(I)$ runs $\mathcal{K}(I) n$ times independently with a restriction such that $g_{2}$ is common for all $p k_{i}$, where $p k_{i}=\left(g_{2}, h_{i}, c_{i}, d_{1 i}, \cdots, d_{k i}\right)$. That is, the encryption keys $p k_{i}$ are not independent of each other while the secret keys $s k_{i}$ are independently chosen. This is possible because $w$ is not a part of $s k_{i}$, where $g_{2}=g_{1}^{w}$.

For $S=\left\{1_{1}, \cdots i_{s}\right\}$, let $M_{i}$ be a plaintext for recipient $i \in S$. Then a ciphertext for $S$ is

$$
C_{S}=\left(u_{1}, u_{2}, e_{i_{1}}, v_{i_{1}}, \cdots, e_{i_{n}}, v_{i_{n}}\right)
$$

such that $u_{1}=g_{1}^{r}, u_{2}=g_{2}^{r}$ and $e_{i}=h_{i}^{r} M_{i}, v_{i}=\left(c_{i} d_{1 i}^{\alpha_{1 i}} \cdots d_{k i}^{\alpha_{k i}}\right)^{r}$, where $r \stackrel{R}{\leftarrow}$ $Z_{p}$ and $\left(\alpha_{1 i}, \cdots, \alpha_{k i}\right)=F\left(u_{1}, u_{2}, e_{i}\right) . T A K E_{i}$ is defined as $\left(u_{1}, u_{2}, e_{i}, v_{i}\right) \leftarrow$ $T A K E_{i}\left(C_{S}\right) . C_{S} \leftarrow T A K E_{S}\left(C_{N}\right)$ is defined naturally.

Note that the size of the ciphertext of our scheme is almost a half of the trivial multi-recipient scheme. We next prove that our scheme is still secure. More precisely, we prove that our scheme is secure in the sense of indistinguishability against chosen ciphertext attack under the DDH assumption.

Lemma 5.1. In the proposed (multi-plaintext,multi-recipient) Cramer-Shoup scheme,

$$
\operatorname{AdvTO}_{\mathcal{C} \mathcal{S}^{n},\left(p, g_{1}\right)}^{n-c a}\left(t, q_{d}\right) \leq 2 \cdot \operatorname{Adv}_{p, g_{1}}^{d d h}\left(t^{\prime}\right)+3 n \epsilon,
$$

where $t^{\prime}=t+O\left(n \cdot q_{d} \cdot T^{e x p}\right)$. 
The proof is similar to that of Theorem 5.1 From lemma 5.1 and lemma 3.1 we obtain the following theorem.

Theorem 5.2. In the proposed (multi-plaintext,multi-recipient) Cramer-Shoup scheme,

$$
\operatorname{Adv}_{\mathcal{C} \mathcal{S}^{n},\left(p, g_{1}\right)}^{n-c c a}\left(t, q_{d}\right) \leq q_{e}\left(2 \cdot \operatorname{Adv}_{p, g_{1}}^{d d h}\left(t^{\prime}\right)+3 n \epsilon\right),
$$

where $t^{\prime}=t+O\left(n \cdot q_{d} \cdot T^{e x p}\right)+O\left(q_{e} n T^{e x p}\right)$.

Comparing with the concrte security of the trivial multi-recipient (basic) Cramer-Shoup scheme given by Bellare et al. [2], we can see that our scheme takes no extra cost fot the "shortened ciphertext" property except negligible factors.

\section{Multi-recipient Hybrid Encryption Scheme}

In this section, we first prove that the (single-plaintext,multi-recipient) hybrid encryption scheme mentioned in Sec1.1 is secure against chosen plaintext attack if the underlying single-recipient public-key scheme is secure in the sense of indistinguishability.

We next present a (single-plaintext,multi-recipient) scheme secure against chosen ciphertext attack with the "shortened ciphertext" property. The underlying single-recipient public-key scheme needs to be secure in the sense of indistinguishability against chosen ciphertext attack. In this sheme, we can use Rabin-SAEP or RSA-SAEP ${ }^{+}$[4] as the underlying single-recipient scheme, for example.

\subsection{IND-CPA Hybrid Scheme}

For a single-recipient public-key encryption scheme $\mathcal{P} \mathcal{E}=(\mathcal{K}, \mathcal{E}, \mathcal{D})$, define a single-recipient hybrid encryption scheme $\mathcal{H}=\left(\mathcal{K}_{H}, \mathcal{E}_{H}, \mathcal{D}_{H}\right)$ as follows. The key generation algorithm is $\mathcal{K}_{H}=\mathcal{K}$. For a plaintext $M$, a ciphertext is

$$
C=\mathcal{E}_{p k}(r) \| M \oplus G(r),
$$

where $p k$ is a public key, $r$ is a random element and $G$ is a pseudorandom generator.

We next define an (single-plaintext,multi-recipient) scheme $\mathcal{H}^{n}=\left(\mathcal{K}_{H}^{n}, \mathcal{E}_{H}^{n}\right.$, $T A K E)$ for $\mathcal{H}$ as follows. The key generation algorithm $\mathcal{K}_{H}^{n}(I)$ runs $\mathcal{K}_{H}(I) n$ times independently, where $I$ is some global information. Let $\left(p k_{i}, s k_{i}\right)$ be the pair of public/secret keys of recipient $i$.

Let $M$ be a plaintext for $S=\left\{1_{1}, \cdots i_{s}\right\}$. Then a ciphertext for $S$ is

$$
C_{S}=\mathcal{E}_{p k_{i_{1}}}(r)\|\cdots\| \mathcal{E}_{p k_{i_{s}}}(r) \| M \oplus G(r),
$$

where $r \stackrel{R}{\leftarrow} Z_{p}$. TAKE $E_{i}$ is defined as $\mathcal{E}_{p k_{i}}(r) \| M \oplus G(r) \leftarrow T A K E_{i}\left(C_{S}\right)$. For $T \subset S \subseteq N, C_{T} \leftarrow T A K E_{T}\left(C_{S}\right)$ is defined naturally. 
It is clear that the size of $C_{S}$ is less than a half of the trivial scheme if $|M|>\left|\mathcal{E}_{p k_{i}}(r)\right|$. We will prove that $\mathcal{H}^{n}$ is secure against chosen plaintext attack if $\mathcal{P} \mathcal{E}$ is secure in the same sense.

Consider that $G$ is a random oracle. Suppose that an adversary makes at most $q_{G}$ queries to the $G$-oracle. Let $r$ be $l$-bits long. Let $\mathcal{P} \mathcal{E}^{n}$ be the trivial multi-recipient scheme for $\mathcal{P} \mathcal{E}$.

Then we first show that $\mathcal{H}^{n}$ is secure if $\mathcal{P} \mathcal{E}^{n}$ is secure against type 0 adversaries.

Lemma 6.1. In the proposed (single-plaintext,multi-recipient) scheme $\mathcal{H}^{n}$,

$$
\operatorname{AdvTO}_{\mathcal{H}^{n}, I}^{n-c p a}(t) \leq \operatorname{AdvTO}_{\mathcal{P} \mathcal{E}^{n}, I}^{n-c p a}\left(t^{\prime}\right)+\frac{q_{G}}{2^{l-3}},
$$

where $t^{\prime}=t+O\left(q_{G}\right)+O(n)$.

A proof will be given in the final paper. For $\mathcal{P} \mathcal{E}^{n}$, the result of Bellare et al. implies that 2 ]

$$
\operatorname{AdvTO}_{\mathcal{P} \mathcal{E}^{n}, I}^{n-\text { cpa }}(t) \leq n \cdot \operatorname{Adv}_{\mathcal{P} \mathcal{E}, I}^{\mathrm{cpa}}\left(t^{\prime}\right)
$$

where $t^{\prime}=t+O(\log (n))$. Therefore, from lemma 3.1, we obtain the following theorem.

Theorem 6.1. In the proposed (single-plaintext,multi-recipient) scheme $\mathcal{H}^{n}$,

$$
\operatorname{Adv}_{\mathcal{H}^{n}, I}^{n-c p a}\left(t, q_{e}\right) \leq q_{e}\left(n \cdot \operatorname{Adv}_{\mathcal{P} \mathcal{E}, I}^{c p a}\left(t^{\prime}\right)+\frac{q_{G}}{2^{l-3}}\right)
$$

where $t^{\prime}=t+O\left(q_{G}\right)+O(n)+O\left(q_{e} T_{n}\right)$, where $T_{n}$ denotes the time to compute a ciphertext $C_{N}$.

\subsection{IND-CCA Hybrid Scheme}

For a single-recipient public-encryption scheme $\mathcal{P} \mathcal{E}=(\mathcal{K}, \mathcal{E}, \mathcal{D})$, define a singlerecipient hybrid encryption scheme $\mathcal{H Y}=\left(\mathcal{K}_{Y}, \mathcal{E}_{Y}, \mathcal{D}_{Y}\right)$ as follows. The key generation algorithm is $\mathcal{K}_{Y}=\mathcal{K}$. For a plaintext $M$, a ciphertext is $C=c_{1}\left\|c_{2}\right\| c_{3}$ with

$$
c_{1}=M \oplus G(r), c_{2}=H(r \| M), c_{3}=\mathcal{E}_{p k}(r),
$$

where $p k$ is a public key, $r$ is a random element, $H$ is a hash function and $G$ is a pseudorandom generator. The decryption algorithm $\mathcal{D}_{Y}$ is defined as

$$
\mathcal{D}_{Y s k}\left(c_{1}\left\|c_{2}\right\| c_{3}\right)= \begin{cases}\text { reject } & \text { if } \mathcal{D}_{s k}\left(c_{3}\right)=\text { reject or } c_{2} \neq H\left(\hat{r} \| c_{1} \oplus G(\hat{r})\right) \\ c_{1} \oplus G(\hat{r}) & \text { otherwise, }\end{cases}
$$

where $s k$ is a secret key and $\hat{r}=\mathcal{D}_{s k}\left(c_{3}\right)$.

Now we define an (single-plaintext,multi-recipient) scheme $\mathcal{H Y}^{n}=\left(\mathcal{K}_{Y}^{n}, \mathcal{E}_{Y}^{n}\right.$, $T A K E)$ for $\mathcal{H} \mathcal{Y}=\left(\mathcal{K}_{Y}, \mathcal{E}_{Y}, \mathcal{D}_{Y}\right)$ as follows. The key generation algorithm $\mathcal{K}_{Y}^{n}(I)$ runs $\mathcal{K}_{Y}(I) n$ times independently, where $I$ is some global information. Let $\left(p k_{i}, s k_{i}\right)$ be the pair of public/secret keys of recipient $i$. 
Let $M$ be a plaintext for $S=\left\{1_{1}, \cdots i_{s}\right\}$. Then a ciphertext for $S$ is

$$
C_{S}=c_{1}\left\|c_{2}\right\| \mathcal{E}_{p k_{i_{1}}}(r)\|\cdots\| \mathcal{E}_{p k_{i_{s}}}(r)
$$

where $r \stackrel{R}{\leftarrow} Z_{p}$. TAKE $E_{i}$ is defined as $c_{1}\left\|c_{2}\right\| \mathcal{E}_{p k_{i}}(r) \leftarrow T A K E_{i}\left(C_{S}\right)$. For $T \subset$ $S \subseteq N, C_{T} \leftarrow T A K E_{T}\left(C_{S}\right)$ is defined naturally.

It is clear that the size of $C_{S}$ is less than a half of the trivial scheme if $\left|c_{1}\right|+\left|c_{2}\right|>\left|\mathcal{E}_{p k_{i}}(r)\right|$. We will prove that $\mathcal{H Y}^{n}$ is secure against chosen ciphertext attack if $\mathcal{P} \mathcal{E}$ is secure in the same sense.

Let $\mathcal{P} \mathcal{E}^{n}$ be the trivial multi-recipient scheme for $\mathcal{P} \mathcal{E}$. We consider that $G$ and $H$ are random oracles. Suppose that an adversary makes at most $q_{G}$ queries to the $G$-oracle, at most $q_{H}$ queries to the $H$-oracle and at most $q_{d}$ queries to each decryption oracle $\mathcal{D}_{s k_{i}}$. Suppose that $r$ is $l$-bits long, $M$ is $k$-bits long, $r \| M$ is $m$-bits long and $H(r \| M)$ be $h$ bits long. Let

$$
\sigma \stackrel{\text { def }}{=} n q_{d}\left(\frac{1}{2^{h-2}}+\frac{1}{2^{k-1}}\right)+5 \frac{q_{G}+q_{H}}{2^{l-2}} .
$$

Then

Lemma 6.2. In the proposed (single-plaintext,multi-recipient) scheme $\mathcal{H Y}^{n}$,

$$
\operatorname{AdvTO}_{\mathcal{H} \mathcal{Y}^{n}, I}^{n-c c a}\left(t, q_{d}\right) \leq 2 \operatorname{AdvTO}_{\mathcal{P} \mathcal{E}^{n}, I}^{n-c c a}\left(t^{\prime}, q_{d}\right)+\sigma,
$$

where $t^{\prime}=t+O\left(q_{d} q_{H}\right)+O\left(q_{G}\right)+O(n)$.

A proof will be given in the final paper. Similarly to Theorem 6.1, we finally have the following Theorem.

Theorem 6.2. In the proposed (single-plaintext,multi-recipient) scheme $\mathcal{H Y}^{n}$,

$$
\operatorname{Adv}_{\mathcal{H} \mathcal{Y}^{n}, I}^{n-c c a}\left(t, q_{e}, q_{d}\right) \leq q_{e}\left(2 n \cdot \operatorname{Adv}_{\mathcal{P} \mathcal{E}, I}^{c c a}\left(t^{\prime}, q_{d}\right)+\sigma\right)
$$

where $t^{\prime}=t+O\left(q_{d} q_{H}\right)+O\left(q_{G}\right)+O(n)+O\left(q_{e} T_{n}\right)$, where $T_{n}$ denotes the time to compute $C_{N}$.

\subsection{Improvement on Multi-recipient ElGamal and Cramer-Shoup}

In our (multi-plaintext,multi-recipient) ElGamal encryption scheme, suppose that $M=M_{i_{1}}=\cdots=M_{i_{s}}$. In this case, let a ciphertext be

$$
\bar{C}=\left(M g^{r}, X_{i_{1}}^{r}, \cdots, X_{i_{s}}^{r}\right) .
$$

This scheme is better than our scheme of Sec4.2 because $M$ is multiplied once. The security is proved similarly. Further, we can consider a hybrid scheme such that

$$
\bar{C}^{\prime}=\left(K g^{r}, X_{i_{1}}^{r}, \cdots, X_{i_{s}}^{r}\right) \| G(K) \oplus M
$$

We can improve our multi-recipient Cramer-Shoup scheme similarly. 


\section{Acknowledgement}

The author thanks Dr. Burt Kaliski for providing many comments, who shepherded the rewriting of the paper.

\section{References}

1. O.Baudron, D.Pointcheval and J.Stern: "Extended Notions of Security for Multicast Public Key Cryptosystems", ICALP '2000 (2000)

2. M.Bellare, A.Boldyreva and S.Micali: "Public-key encryption in a multi-recipient setting: Security proofs and improvements", Advances in Cryptology - Eurocrypt'2000 Proceedings, Lecture Notes in Computer Science Vol.1807, Springer Verlag, pp.259-274 (2000)

3. M.Bellare and P.Rogaway: "Random oracles are practical: A paradigm for designing efficient protocols", Proc. of the 1st CCS, pp.62-73, ACM Press, New York, 1993.

(http://www-cse.ucsd.edu/users/mihir/crypto2k)

4. D.Boneh: "Simplified OAEP for the RSA and Rabin Functions", Advances in Cryptology - Crypto'2001 Proceedings, Lecture Notes in Computer Science Vol.2139, Springer Verlag, pp.275-291 (2001)

5. D. Boneh and M. Franklin: "An efficient public key traitor tracing scheme", Advances in Cryptology - Crypto'99 Proceedings, Lecture Notes in Computer Science Vol.1666, Springer Verlag, pp.338-353 (1999)

6. B. Chor, A. Fiat, and M. Naor, B. Pinkas: "Tracing traitors", IEEE Trans. on IT, vol.46, no.3, pages 893-910 (2000).

7. D.Coppersmith: "Finding a small root of a univariate modular equation", Advances in Cryptology - Eurocrypt'96 Proceedings, Lecture Notes in Computer Science Vol.1070, Springer Verlag, pp.155-165 (1996)

8. D.Coppersmith: "Small solutions to polynomial equations, and low exponent RSA vulnerabilities", Journal of Cryptology, 10, pp.233-260 (1997)

9. R.Cramer and V.Shoup: "A practical public key cryptosystem provably secure against adaptive chosen ciphertext attack", Advances in Cryptology - Crypto'98 Proceedings, Lecture Notes in Computer Science Vol.1462, Springer Verlag, pp.13$25(1998)$

10. S.Goldwasser and S.Micali : "Probabilistic encryption", Journal Computer and System Sciences, vol.28, pp.270-299 (1984).

11. J.Hastad : "Solving simultaneous modular equations of low degree", SIAM Journal of Computing, vol.17, pp.336-341 (1988).

12. K. Kurosawa and Y. Desmedt: Optimum traitor tracing and asymmetric schemes with arbiter. Advances in Cryptology - Eurocrypt'98, Lecture Notes in Computer Science \#1403, Springer Verlag (1999) 145-157

13. K.Kurosawa and T.Yoshida: "Linear code implies public-key traitor tracing", PKC'02 (this proceedings)

14. M.Naor and O.Reingold : "Number theoretic constructions of efficient pseudorandom functions", FOCS'97, pp.458-467 (1997).

15. M.Stadler: "Publicly verifiable secret sharing", Advances in Cryptology - Eurocrypt'96 Proceedings, Lecture Notes in Computer Science Vol.1070, Springer Verlag, pp.190-199 (1996)

16. Y.Zheng amd J.Seberry: "Practical approaches to attaining security against adaptively chosen ciphertext attacks", Advances in Cryptology - Crypto'92 Proceedings, Lecture Notes in Computer Science Vol.740, Springer Verlag, pp.292-304 (1992) 


\section{A Proof of Lemma 3.1}

We show a proof for (multi-plaintext, multi-recipient) schemes against chosenplaintext attack. The proofs for the other cases are similar. Let $B$ be an adversary which has time-complexity $t$ and makes at most $q_{e}$ queries. We will design an type 0 adversary $D_{B}$ with time-complexity at most $t^{\prime}$.

Similar to [2], we consider a hybrid experiment with a parameter $l$ such that $0 \leq l \leq q_{e}$ as follows.

Experiment-l: Let the $i$-th query of $B$ be $\left(S, \underline{M}_{S}^{0}, \underline{M}_{S}^{1}\right)$. If $i \leq l$, then $\mathcal{E}_{p k}$ returns $\mathcal{E}_{\underline{p k}}\left(S, \underline{M}_{S}^{1}\right)$. Otherwise, it returns $\mathcal{E}_{\underline{p k}}\left(S, \underline{M}_{S}^{0}\right)$.

Let

$$
p_{l} \stackrel{\text { def }}{=} \operatorname{Pr}[\tilde{b}=0 \text { in Experiment- } l] .
$$

Then it is easy to see that

$$
\operatorname{Adv}_{\mathcal{P} \mathcal{E}^{n}, I}^{n-\mathrm{cpa}}(B)=p_{0}-p_{q_{e}} .
$$

Next our $D_{B}$ works as follows. On input $(I, p k), D_{B}$ chooses $l$ randomly such that $1 \leq l \leq q_{e}$. It runs $B$ by giving $(I, p k) \overline{\text { to }} B$. Let the $i$-th query of $B$ be $\left(S, \underline{M}_{S}^{0}, \underline{M}_{S}^{1}\right)$.

1. If $i<l$, then $D_{B}$ returns $C_{S}^{1} \stackrel{R}{\leftarrow} \mathcal{E}_{\underline{p k}}\left(S, \underline{M}_{S}^{1}\right)$.

2. If $i>l$, then $D_{B}$ returns $C_{S}^{0} \stackrel{R}{\leftarrow} \mathcal{E}_{p k}\left(S, \underline{M}_{S}^{0}\right)$.

3. If $i=l$, then $D_{B}$ queries $\left(N, \underline{M}_{N}^{0}, \underline{M}_{N}^{1}\right)$ to his encryption oracles, where $\underline{M}_{S}^{0}$ and $\underline{M}_{S}^{1}$ are naturally embedded in $\underline{M}_{N}^{0}$ and $\underline{M}_{N}^{1}$, respectively. The oracle returns $C_{N}^{b} \stackrel{R}{\leftarrow} \mathcal{E}_{\underline{p k}}\left(N, \underline{M}_{N}^{b}\right)$ to $D_{B} . D_{B}$ finally gives $C_{S}^{b}=T A K E_{S}\left(C_{N}^{b}\right)$ to $B$.

Suppose that $B$ outputs $\tilde{b}$ finally. Then $D_{B}$ outputs $\tilde{b}$.

Now we can see that

$$
\begin{aligned}
& \operatorname{Pr}(\tilde{b}=0 \mid b=0)=\left(p_{0}+\cdots p_{q_{e}-1}\right) / q_{e} \\
& \operatorname{Pr}(\tilde{b}=0 \mid b=1)=\left(p_{1}+\cdots p_{q_{e}}\right) / q_{e}
\end{aligned}
$$

because $l$ is randomly chosen. Therefore,

$$
\operatorname{Adv}_{\mathcal{P} \mathcal{E}^{n}, I}^{n-\mathrm{cpa}}\left(D_{B}\right)=\left(p_{0}-p_{q_{e}}\right) / q_{e}=\operatorname{Adv}_{\mathcal{P E}^{n}, I}^{n-c^{n} a}(B) / q_{e}
$$

Hence

$$
\operatorname{Adv}_{\mathcal{P} \mathcal{E}^{n}, I}^{n-\text { cpa }}(B)=q_{e} \cdot \operatorname{Adv}_{\mathcal{P} \mathcal{E}^{n}, I}^{n-\text { cpa }}\left(D_{B}\right) .
$$

By taking the maximum, we obtain that

$$
\operatorname{Adv}_{\mathcal{P E}^{n}, I}^{n \text {-cpa }}\left(t, q_{e}\right) \leq q_{e} \cdot \operatorname{AdvT0}_{\mathcal{P} \mathcal{E}^{n}, I}^{n-\mathrm{cpa}}\left(t^{\prime}\right)
$$

Finally, the overhead of $D_{B}$ is to pick the random number $l$ and execute some conditional statements. It is $O\left(q_{e} \cdot T_{n}\right)$. 


\section{B Proof of Lemma 4.1}

By extending the result of Stadler [15, in the proof of Proposition 1] and Naor and Reingold [14, lemma 3.2], Bellare et al. proved the following proposition [2].

Proposition B.1. 20 There is a probabilistic algorithm $R$ such that on input $g^{a}, g^{b}, g^{c}, R$ outputs $g^{b^{\prime}}, g^{c^{\prime}}$, where $b^{\prime}$ is random and

$$
c^{\prime}= \begin{cases}a b^{\prime} \bmod p & \text { if } c=a b \bmod p \\ r a n d o m & \text { if } c \neq a b \bmod p\end{cases}
$$

$R$ runs in $O\left(T^{\exp }\right)$ time.

Now we show a proof of lemma 4.1 Let $B$ be a type 0 adversary attacking the proposed scheme with time-complexity at most $t$. We will design an adversary $D_{B}$ for the DDH problem, where $D_{B}$ has time complexity at most $t^{\prime}$.

Let the input to $D_{B}$ be $g^{r}, g^{x}, g^{z}$. $D_{B}$ runs $R$ of Proposition B.1 $n$ times independently on input $\left(g^{r}, g^{x}, g^{z}\right)$. Then $R$ outputs $X_{1}=g^{x_{1}}, \cdots, X_{n}=g^{x_{n}}$ and $Z_{1}=g^{z_{1}}, \cdots, Z_{n}=g^{z_{n}}$, where $x_{1}, \cdots, x_{n}$ are random and

$$
z_{i}= \begin{cases}r x_{i} \bmod p & \text { if } z=r x \bmod p \\ \text { random } & \text { if } z \neq r x \bmod p\end{cases}
$$

$D_{B}$ gives $X_{1}, \cdots, X_{n}$ to $B$ as $n$ public keys and runs $B$. Suppose that $B$ queries $\left(M_{0,1}, \ldots, M_{0, n}\right)$ and $\left(M_{1,1}, \ldots, M_{1, n}\right)$ in the find stage. Then $D_{B}$ chooses a random bit $b$ and gives $\tilde{C}=\left(g^{r}, M_{b, 1} \cdot Z_{1}, \cdots, M_{b, n} \cdot Z_{n}\right)$ to $B$ as a challenge ciphertext. Suppose that $B$ outputs $\tilde{b}$ in the guess stage. Finally, $D_{B}$ outputs $b \oplus \tilde{b}$.

First suppose that $\left(g^{r}, g^{x}, g^{z}\right) \in D H$. Then $\tilde{C}$ is a legal ciphertext. Therefore, as shown in eq.(3), we have

$$
\operatorname{Pr}\left(D_{B} \text { outputs } 0\right)=\operatorname{Pr}(\tilde{b}=b)=\frac{1}{2}+\frac{1}{2} \operatorname{Adv}_{\mathcal{E} \mathcal{G}^{n},(p, g)}^{n \text {-ca }}(B) .
$$

Next suppose that $\left(g^{r}, g^{x}, g^{z}\right) \in R A$. If $z \neq r x$, then $Z_{1}, \cdots, Z_{n}$ are random and $\operatorname{Pr}(\tilde{b}=b)=1 / 2$. Hence, we have

$$
\begin{aligned}
\operatorname{Pr}\left(D_{B} \text { outputs } 0\right) & =\operatorname{Pr}(\tilde{b}=b) \\
& \leq \frac{1}{2}\left(1-\frac{1}{p}\right)+\frac{1}{p}=\frac{1}{2}+\frac{1}{2 p}
\end{aligned}
$$

From eq.(13) and eq.(14), we have

$$
\begin{aligned}
\operatorname{Adv}_{p, g}^{\mathrm{ddh}}\left(D_{B}\right) & \geq \frac{1}{2} \operatorname{Adv}_{\mathcal{E} \mathcal{G}^{n},(p, g)}^{n-\mathrm{cpa}}(B)-\frac{1}{2 p} \\
\operatorname{Adv}_{\mathcal{E} \mathcal{G}^{n},(p, g)}^{n-\text { cpa }}(B) & \leq 2 \operatorname{Adv}_{p, g}^{\mathrm{ddh}}\left(D_{B}\right)+\frac{1}{p}
\end{aligned}
$$

By taking the maximum, we have

$$
\operatorname{AdvTO}_{\mathcal{E} \mathcal{G}^{n},(p, g)}^{n \text {-cpa }}(t) \leq 2 \cdot \operatorname{Adv}_{p, g}^{\mathrm{ddh}}\left(t^{\prime}\right)+\frac{1}{p} .
$$

It is easy to see that $t^{\prime}=t+O\left(n \cdot T^{\exp }\right)$. 